\title{
Study the impact of diabetes outcomes with covid 19 in Rewa
}

\author{
Dr. Shivani Awasthi
}

Department of Biochemistry, A.P.S.U., Rewa, (M.P.) India

Received: 20-11-2021 / Revised Accepted: 30-12-2021 / Published: 02-01-2022

\begin{abstract}
Eighteen months into the severe acute respiratory syndrome corona virus 2 (SARS-CoV-2) (corona virus disease 2019 [COVID-19]) pandemic, epidemiologic studies show that diabetes is a vital contributor to severe COVID-19 morbidity, and, conversely, COVID-19 has had a devastating effect on the population with diabetes. Most cases that have been admitted have been mild to moderate since the early stages of the outbreak. However, there has been an enhance in the number of severe cases and deaths as a outcome of COVID-19, which creates it imperative to evaluate the risk factors for progression of the disease. Current evidence from China and the US recommends that co- morbidities such as hypertension, diabetes, obesity, chronic obstructive pulmonary disease (COPD) and cerebro-vascular disease increase the risk of severity and death from COVID-19. However, there are significant differences in demographic patterns and disease trends between high-income and low- and middle-income countries. COVID-19 infection disrupts glucose regulation, rendering glycemic control difficult and necessitating particularly careful management in patients with diabetes.
\end{abstract}

Key words: diabetes, morbidity, mild, moderate, severe, co-morbidity, cerebrovascular, glycemic control, acute respiratory

\section{INTRODUCTION}

The coronavirus disease 2019 (COVID-19) pandemic has infected .22 .7 million and killed. 795,000 people world-wide, as of 21 August 2020 (1). Normally COVID-19 infection is caused by severe acute respiratory syndrome corona virus 2 (SARS-CoV-2), a single-stranded RNA b-corona virus (2). Patients with diabetes are very susceptible to adverse results and complications of COVID-19 infection (3). The COVID-19 pandemic is superimposing on the pre-existing diabetes pandemic to make large and significantly vulnerable populations of patients with COVID-19 and diabetes. Other comorbid situations frequent in patients with type 2 diabetes, e.g., cardiovascular disease (CVD) and obesity, also predispose COVID-19 patients to adverse clinical results (4).

Corona viruses are a family of viruses with a genome size of around 26 to 32 kilo bases and a size of $80-220 \mathrm{~nm}$ in diameter, making them the largest among RNA viruses $(5,6)$. They are surrounded and enveloped by a outer layer which is made by fatty acid and generally have spherical shapes with a crown or "corona" of club-shaped spikes on their surface. The spikes of corona virus are responsible for the virus-receptor binding in

Address for Correspondence: Dr. Shivani Awasthi, Department of Biochemistry, A.P.S.U., Rewa, (M.P.) India; Email: shivaniawasthi786@gmail.com

How to Cite this Article: Shivani Awasthi. Study the impact of diabetes outcomes with covid 19 in Rewa. World J Pharm Sci 2022; 10(01): 136-143; https://doi.org/10.54037/WJPS.2022.100115 
cell surface (7). Arthur et al. in the 1930s were the first to report a case of acute respiratory infection in chickens and proposed that it may be related to a specific type of viruses (8). Later, Fred and coworkers in 1937 isolated and cultivated the corona virus for the first time in the lab (9). In the 1960s, the first human corona virus was found from a boy who had a new type of cold(10,11). Corona virus gains entry by binding to the cell surface receptors via the $\mathrm{S}$ protein (spike), which is then cleaved into two functional subunits known as S1 and S2 (12). Upon binding of $\mathrm{S} 1$ to the specific receptor, a conformational change is triggered in the S2 subunit, followed by viral release into the cytoplasm after some sequential molecular steps (12).

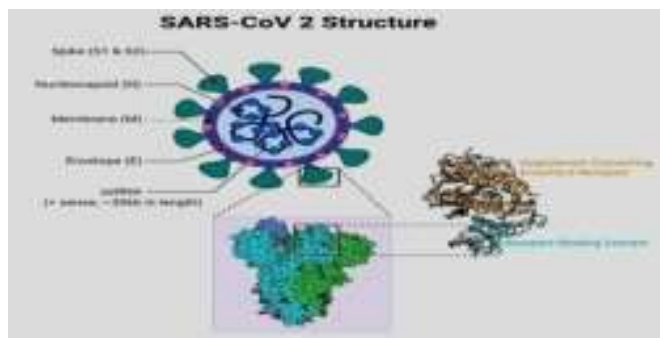

Picture 1: Structure of SARS -COV-2 Virus .

Until today, seven types of viruses from this family are discovered to infect humans which are SARS$\mathrm{CoV}$, MERS-CoV (Middle East respiratory syndrome corona virus), SARS-CoV-2, HKU1 (Human corona virus 1), NL63 (Human corona virus NL63), OC43 (Human corona virus OC43), and 229E (Human corona virus 229E) (13). While the first three viruses are connected to severe respiratory infections, others normally cause mild upper respiratory symptoms (13). We know that SARS-CoV-2 is a single-stranded and an significant member of the corona virus family which was just discovered in late 2019 as responsible for the COVID-19 pandemic .It was firstly reported in Wuhan, the capital of China's Hubei province $(14,15)$. COVID-19 is now quickly spreading in more than 200 countries, and as of May 10, 2020, there are 4.02 million confirmed cases of COVID-19 and 279,000 casualties reported worldwide (16). Fever, fatigue, cough, shortness of breathing, chest pain, and loss of smell are the general symptoms of COVID-19 (17). However, in severe cases, it can cause severe lower respiratory tract symptoms and low oxygen saturation in the blood similar to acute respiratory distress syndrome and will need mechanical ventilation (18). The World Health Organization (WHO) confirmed that the COVID-19 outbreak is a Public Health Emergency of International Concern on January 30, 2020, and a pandemic on 11 March 2020 (19). At present, we have no exact vaccine or antiviral treatment available to manage COVID-19. The primary strategy for managing COVID-19 currently is only symptomatic treatments and supportive care in addition to isolation, and experimental therapies (20).

As stated earlier, DM increases the problems of COVID-19 and the risk of COVID-19 related death (21). Present evidence shows that patients with DM are more likely to experience severe symptoms and problems than patients without DM due to COVID19 (21). One hypothesis is that hyperglycemia facilitates the virus entry into the cells since ACE2 and virus both need glucose for their function (22). Although to understand the exact interactions between COVID-19 and DM needs more research, we have reviewed the potential molecular mechanisms involved from a cell biology point of view. The death rate due to COVID-19 in India is very low (i.e. $2.57 \%$ ) and interestingly most (nearly $73 \%$ ) of deaths are being observed in those having comorbidities (23). Among all, diabetes discovered to pose a particular adverse risk for COVID-19 infection (24). Uncontrolled diabetes compromises innate immunity, the first line of defence against SARS-CoV-2 (25) India harbors 77 million diabetes patients, which creates it the second most affected nation in the world, after China. The number is projected to produce 134 million by 2045 as per the International Diabetes Federation (26). By virtue of being with a elevated prevalence of diabetes and a hasty upsurge in COVID-19 cases during the unlocking phase, and with their overlapping on morbidity and death of people, it is necessary to study the impact of COVID-19 on diabetic care in India and helps improvise the preparedness for present and future thereat imposed by COVID-19.

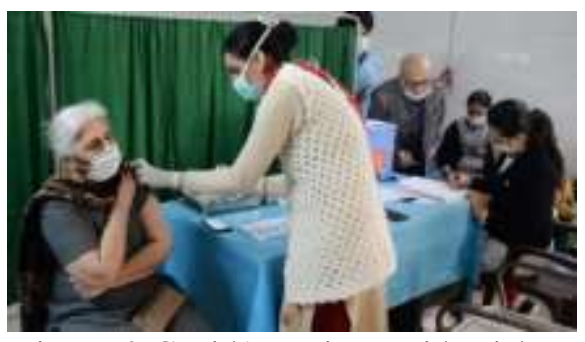

Picture 2-Covid19 patients with Diabetes.

Since the outbreak of COVID-19, some studies have been conducted to examine the relationship between diabetes and COVID-19 (27). However, their sample sizes were relatively tiny, and they did not clarify whether diabetes was a predictor of poor clinical results and higher death of COVID-19. The aim of this study, to investigate the association between diabetes as a comorbidity and negative clinical courses and outcomes of COVID-19 in a sample of patients. The findings are expected to inform clinical treatment for patients with both diseases and COVID-19. 


\section{MATERIALS AND METHODS}

Study Design and Participants: This observational study was performed on 475 patients diagnosed with COVID-19 at APS University. After excluding cases without information about the history of diabetes, the final sample included 250 patients. Data were collected on admission or during hospitalization by attending physicians and documented in the form of electronic medical records. This study was performed between 20 January 2021 to 28 March 2021.

Data Collection: We collected data of 475 patients from their medical records. These data included age, sex, comorbidities, levels of illness severity (mild, severe, and critical), signs and symptoms, treatment options (i.e., antiviral therapy, antibiotic therapy) laboratory findings.

Statistical Analysis: Normally distributed continuous variables are expressed as means \pm standard deviations. Meanwhile, categorical variables are explained as frequencies and percentages. The independent t-test or MannWhitney U-test was performed to compare continuous variables between the group of patients with diabetes (Group 2) and that of patients without diabetes (Group 1). The Kaplan-Meier survival curves were performed to explore the prognosis of patients with and without diabetes. Two-sided $\mathrm{P}<$ 0.05 was considered as statistically significant, and all statistical analyses were performed using Prism (version 3 for Windows).

Observation: The present study entitled, To Study the impact of diabetes results with covid 19 in Rewa was undertaken in the department of Biochemistry APS University REWA (M.P). From 20 January 2021 to 28 March 2021.Study samples, including 250 patients. Following were the observations.

Table 1-Demographics and clinical characteristics of patients with COVID-19

\begin{tabular}{|c|c|c|c|}
\hline Covariate & Group $1(150)$ & Group 2(100) & $\mathrm{P}$-value \\
\hline Age, year & $64.31 \pm 27.81$ & $209 \pm 43.48$ & $\mathrm{P}<0.0001$ \\
\hline \multicolumn{4}{|l|}{ Sex } \\
\hline Male & $90(60 \%)$ & $54(54 \%)$ & 0.7112 \\
\hline Female & $60(40 \%)$ & $46(46 \%)$ & $\mathrm{P}<0.0001$ \\
\hline \multicolumn{4}{|l|}{ Comorbidity } \\
\hline Cardiovascular disease & $75(50 \%)$ & $68(68 \%)$ & $\mathrm{P}<0.0001$ \\
\hline Pulmonary disease & $82(54.6 \%)$ & $5(5 \%)$ & 0.0002 \\
\hline Malignancy & $41(27.33 \%)$ & $12(12 \%)$ & $\mathrm{P}<0.0001$ \\
\hline Nervous system disease & $30(20 \%)$ & $20(20 \%)$ & $\mathrm{P}<0.0001$ \\
\hline Digestive system disease & $24(16 \%)$ & $5(5 \%)$ & 0.0006 \\
\hline \multicolumn{4}{|l|}{ The highest level of severity } \\
\hline Mild and general & $38(25.33 \%)$ & $30(30 \%)$ & $\mathrm{P}<0.0001$ \\
\hline Severe & $60(40 \%)$ & $45(45 \%)$ & $\mathrm{P}<0.0001$ \\
\hline Critical & $52(34.66 \%)$ & $60(60 \%)$ & $\mathrm{P}<0.0001$ \\
\hline \multicolumn{4}{|c|}{ Symptoms when admitted to the hospital } \\
\hline Fever or Myalgia & $51(34 \%)$ & $63(63 \%)$ & $\mathrm{P}<0.0001$ \\
\hline Respiratory system symptoms & $56(37.33 \%)$ & $73(73 \%)$ & $\mathrm{P}<0.0001$ \\
\hline Nervous system symptoms & $15(10 \%)$ & $7(7 \%)$ & 0.0002 \\
\hline Antiviral therapy & $125((83.33 \%)$ & $73(73 \%)$ & $\mathrm{P}<0.0001$ \\
\hline Antibiotic therapy & $130(86.66 \%)$ & $95(95 \%)$ & $\mathrm{P}<0.0001$ \\
\hline The appliance of Vitamin C & $135((90 \%)$ & $90(90 \%)$ & $\mathrm{P}<0.0001$ \\
\hline Use of corticosteroid & $120(80 \%)$ & $11(11 \%)$ & $\mathrm{P}<0.0001$ \\
\hline Deaths & $4(2.66 \%)$ & $12(12 \%)$ & $\mathrm{P}<0.0001$ \\
\hline
\end{tabular}


Shivani, World J Pharm Sci 2022; 10(01): 136-143

Table -2 Laboratory results in the COVID-19 patients with or without diabetes.

\begin{tabular}{|c|c|c|c|}
\hline Covariate & Group $1 *(n=150)$ & Group 2 \# $(n=100)$ & P- Value \\
\hline Interleukin-6, pg/ml & $0.6690(0.3111$ to 1.455$)$ & $0.5118(0.2271$ to 1.002$)$ & 0.0086 \\
\hline Procalcitonin, $\mathrm{ng} / \mathrm{mL}$ & $1.105(0.4858$ to 2.515$)$ & $0.7425(0.2556$ to 2.046$)$ & 0.5682 \\
\hline Alanine aminotransferase, U/L & $1.252(0.5743$ to 2.716$)$ & $0.5766(0.1707$ to 1.555$)$ & 0.2733 \\
\hline Aspartate aminotransferase, U/L & $1.485(0.6827$ to 3.193$)$ & $1.219(0.4894$ to 3.011$)$ & 0.8869 \\
\hline Albumin, $g / L$ & $1.448(0.6654$ to 3.115$)$ & $0.9513(0.3623$ to 2.487$)$ & 0.6320 \\
\hline Creatine kinase, U/L & $1.631(0.7501$ to 3.508$)$ & $1.239(0.4963$ to 3.078$)$ & 0.7992 \\
\hline Lactate dehydrogenase, U/L & $1.729(0.7959$ to 3.729$)$ & $1.164(0.4602$ to 2.957$)$ & 0.6734 \\
\hline Total bilirubin, $\mu \mathrm{mol} / \mathrm{L}$ & $1.966(0.9270$ to 4.292$)$ & $1.149(0.4763$ to 2.779$)$ & 0.4312 \\
\hline Creatinine, $\mu \mathrm{mol} / \mathrm{L}$ & $2.280(1.063$ to 5.096$)$ & $1.194(0.4969$ to 2.8840 & 0.3324 \\
\hline $\mathrm{BUN}, \mathrm{mmol} / \mathrm{L}$ & $2.425(1.134$ to 5.464$)$ & $1.374(0.5559$ to 3.358$)$ & 0.4063 \\
\hline Prothrombin time, $\mathrm{s}$ & $1.632(0.8168$ to 3.432$)$ & $1.019(0.4290$ to 2.425$)$ & 0.2792 \\
\hline $\begin{array}{l}\text { Activated partial thromboplastin } \\
\text { time, } s\end{array}$ & $2.269(1.057$ to 5.068$)$ & $1.275(0.5341$ to 3.053$)$ & 0.4076 \\
\hline Fibrinogen, $\mathrm{g} / \mathrm{L}$ & $2.271(1.058$ to 5.073$)$ & $0.9127(0.3325$ to 2.455$)$ & 0.2069 \\
\hline D-dimer, ng/mL & 2.191 (1.018 to 4.857$)$ & $1.160(0.4584$ to 2.953$)$ & 0.4255 \\
\hline White blood cell count, $\times 109 / \mathrm{L}$ & $2.765(1.322$ to 6.561$)$ & $1.619(0.6610$ to 3.833$)$ & 0.5224 \\
\hline Neutrophil count, $\times 109 / \mathrm{L}$ & $2.724(1.299$ to 6.423$)$ & $1.720(0.7038$ to 4.060$)$ & 0.6470 \\
\hline Lymphocyte count, $\times 109 / \mathrm{L}$ & $2.553(1.160$ to 6.002$)$ & $2.014(0.7733$ to 4.749$)$ & 0.9216 \\
\hline Monocyte count, $\times 109 / \mathrm{L}$ & $2.065(0.9852$ to 4.707$)$ & $1.081(0.4545$ to 2.588$)$ & 0.2369 \\
\hline Red blood cell count, $\times 109 / \mathrm{L}$ & $1.994(0.9598$ to 4.393$)$ & $0.7531(0.3106$ to 1.752$)$ & 0.0319 \\
\hline Hemoglobin, g/L & $2.290(1.094$ to 5.183$)$ & $1.220(0.5106$ to 2.913$)$ & 0.3142 \\
\hline
\end{tabular}

*Group 1 for patients without diabetes.

\# Group 2 for patients with diabetes

Figure 1 - The distribution of the number of deaths by the level of illness severity in the Non DM group 1 and the DM group 2 .

\section{Distribution of deaths}

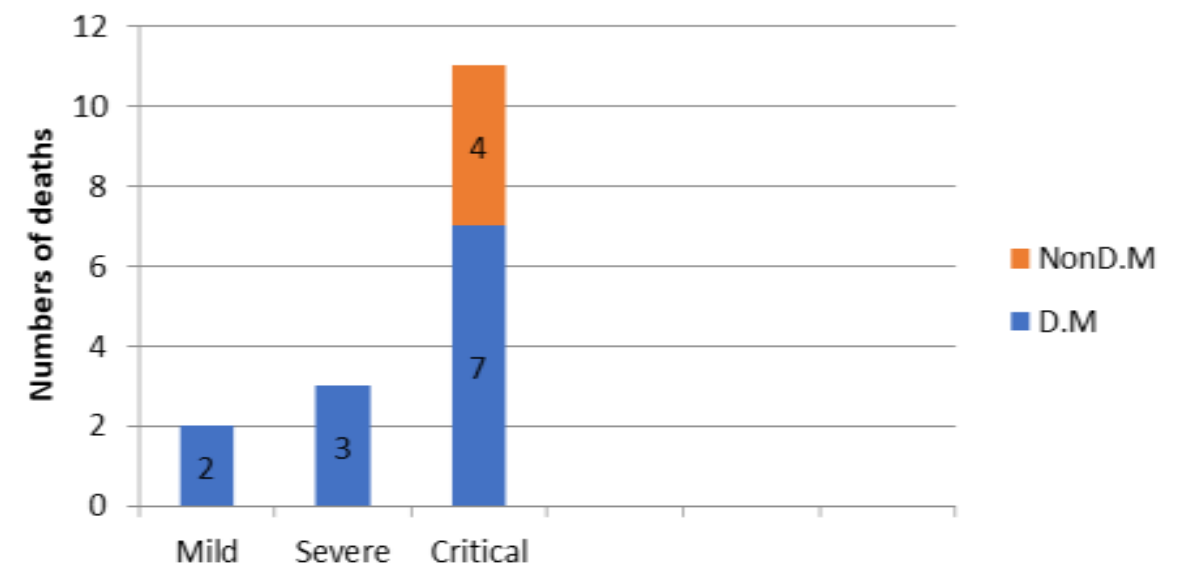


Figure 2 - Covid 19 Comorbidities Grouped by Sex

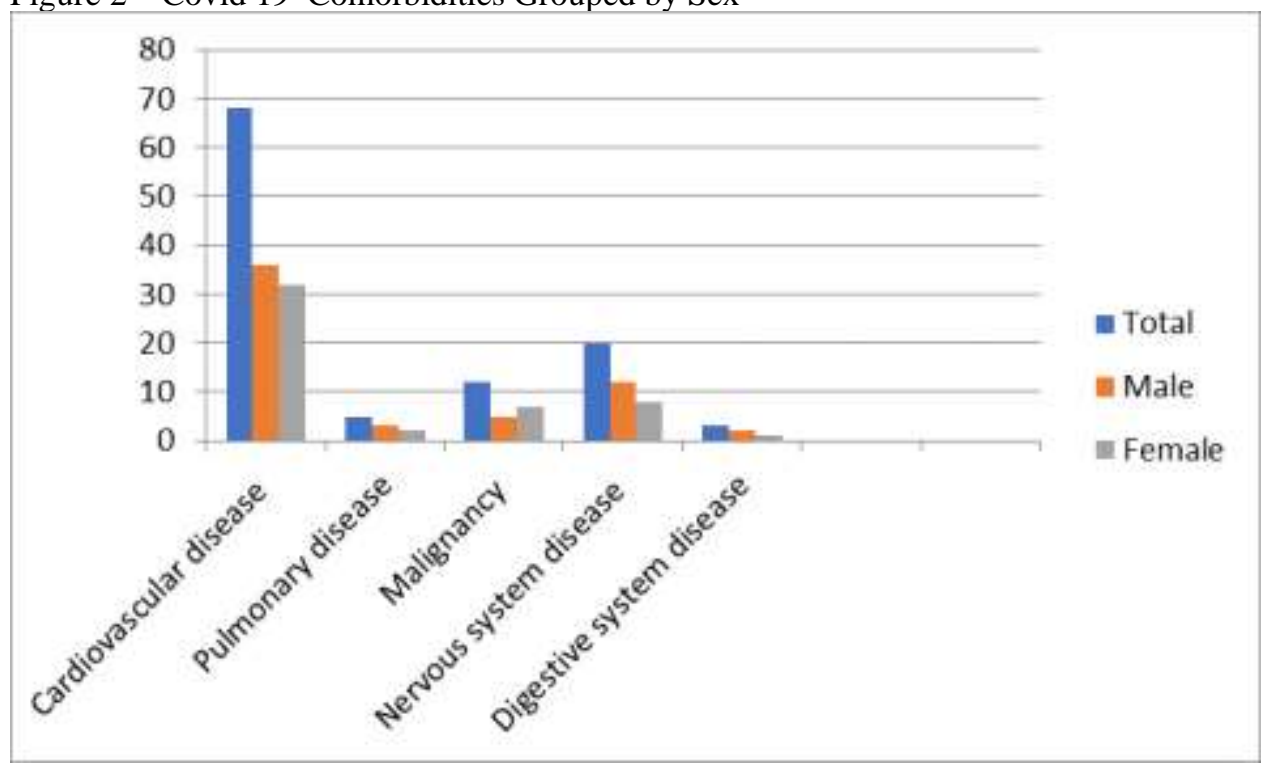

\section{RESULTS}

Table- 1 Shows that Group 2 consisted of 100 patients, whereas Group 1 had 150 patients. The two groups, men's showed no differences in gender distribution $(p=0.7112)$ while women's shows significant difference between two groups $(\mathrm{P}<0.0001)$, means of age $(\mathrm{P}<0.0001)$, and the number of patients with cardiovascular disease, malignancy, and nervous system disease, (all $\mathrm{P}<0.0001)$. However, they showed a not significant difference in the number of patients with pulmonary disease $(\mathrm{P}=0.0002)$ or digestive system disease $(\mathrm{P}=0.0006)$.

The percentages of patients with diabetes among the severe and critical COVID-19 cases were higher than among the mild cases $(45 \%, 60 \%$ vs. $30 \%, \mathrm{P}<0.0001)$. The mortality rate was $12 \%(\mathrm{n}=$ 12) in Group 2 and 2.66\% $(\mathrm{n}=4)$ in Group $1(\mathrm{P}<$ $0.0001)$.

Table 2- In Kaplan Meier survival curves was used to access the association between the clinical factors and the severe clinical events. The Procalcitonin, Alanine aminotransferase, Aspartate aminotransferase, Albumin, Creatine kinase, Lactate dehydrogenase, Creatinine, BUN, Prothrombin, Activated partial, thromboplastin time, Fibrinogen, Neutrophil count, Lymphocyte count, Monocyte count, Hemoglobin were not significantly associated with Group 1 and Group 2 patients. Whereas IL -6 and R.B.C count was significantly associated with Group 1 and Group 2 patients. Figure 1- Shows the distribution of the number of deaths by the level of illness severity in both groups. There were 7critical COVID-19 cases in Group 2, and 4 cases in Group 1. Figure 2- shown that more male than female patients with comorbidities and diabetes.

\section{DISCUSSION}

The prevalence of diabetes mellitus is anticipated to increase substantially during the next decades worldwide and considered to be major reason of human deaths (28). People with diabetes are very susceptible to certain infectious diseases, such as staphylococcus aureus and mycobacterium tuberculosis, possibly because of their dysregulated immune system $(29,30)$. During the outbreak of SARS in 2003 in Guangzhou, Yang et al. Reported that plasma glucose levels and diabetes were independent predictors for death and morbidity, and metabolic control might improve the prognosis in patients with SARS (31). Recently, COVID-19 has been a focal topic of research, and several researches have focused on diabetes as a predictor of clinical course and prognosis of COVID-19 cases (32). Certain studies found that diabetes negatively affected medical complications, including mortality, in COVID-19 cases (33). In my study, collected data with a large sample. I found that COVID-19 patients with DM were older and the proportion of patients with cardiovascular disease were higher among COVID-19 patients with DM than COVID-19 patients without DM. However, the latter had a higher proportion of pulmonary disease as comorbidities than the former. Furthermore, Alanine aminotransferase, aspartate aminotransferase (AST) and Neutrophil count, were lower in COVID-19 patients with diabetes than those without disease. My studies similar to study of (34). Severe or critical COVID19 cases were more common among patients with diabetes than those did not suffer from diabetes. This result of my study similar to the study of (34). 
A systematic review by Huang et al. shown that diabetes was associated with mortality, severity, acute respiratory distress syndrome, and disease progression in patients with COVID- 19 (35). Having measured the association between diabetes with the severity of COVID-19, Wu et al. revealed the proportion of diabetes as a comorbidity among severe COVID-19 cases was significantly higher than that among mild cases (36). Similarly, my data supported that the proportion of severe or critical COVID-19 cases among patients with diabetes was higher than that among those without diabetes. This means the clinical course of COVID-19 in patients with diabetes may be more severe than that in those without diabetes. At present, the mechanisms behind this phenomenon remain unknown; however, high glucose levels may play a certain role in the impaired antibacterial neutrophil function and complications caused by chronic diabetes (28). In addition, the comorbidity with cardiovascular diseases, such as ischemic heart disease and heart failure, was reported to have an association with higher death due to COVID-19 (20). In my study population, $68 \%$ of patients with diabetes suffered from cardiovascular diseases, higher than that of those without diabetes. This may partly explain that the group of patients with diabetes had a higher proportion of COVID-19 cases in severe or critical conditions than the group of those without diabetes.

Figure 1- Shows the distribution of the number of deaths by the level of illness severity in both groups. There were 7 critical COVID-19 cases in Group 2, and 4 cases in Group 1, this study resemble to the study of (34). Figure 2- Shows that more male than female patients with comorbidities and diabetes this finding similar to the study of (38). My study has some limitations that require to be addressed. First, the retrospective, nonrandomized nature led to sample heterogeneity. Second, we were not able to collect and analyze the characteristic data of patients with diabetes, such as the type of diabetes, glucose level, HbA1c and treatment options for diabetes due to the time limitation; hence, we could not analyze the data on anti-DM treatment although they could affect the clinical course and treatment result of COVID-19. Third, we did not observe the relative mechanism behind the effect of diabetes on COVID-19 in this study.

\section{Conclusion}

This study describes the outcomes of patients with diabetes in a large sample of COVID-19 cases. As the COVID-19 pandemic evolves, it is crucial to understand risk factors for disease development and my study helps to fill gaps that currently exist for Indian populations. It is recommended that public enlightenment should be emphatic about high-risk diabetes and covid-19. Those identifications through screening be done to identify undiagnosed cases. Persons with diabetes should be encouraged to utilize all recommended protective measures and seek prompt care for COVID-19. Furthermore, specific guidelines should be developed for home-based and hospital management of COVID-19 patients with diabetes, especially in the India context so that targeted care can be provided to minimize deaths.

\section{REFERENCES}

1. COVID-19 dashboard by the Center for Systems Science and Engineering (CSSE) at Johns Hopkins University (JHU). Accessed 2020.

2. Jin Y, Yang H, Ji W, et al. Virology, epidemiology, pathogenesis, and control of COVID-19. Viruses 2020;12:372

3. Zhu L, She ZG, Cheng $X$, et al. Association of blood glucose control and outcomes in patients with COVID-19 and pre-existing type 2 diabetes. Cell Metab 2020;31:1068-1077.e3

4. Williamson EJ, Walker AJ, Bhaskaran K, et al. Factors associated with COVID19-related death using Open SAFELY. Nature 2020;2020:430-436

5. P. C. Y. Woo, Y. Huang, S. K. P. Lau, and K. Y. Yuen, "Corona virus genomics and bioinformatics analysis," Viruses ,2010, vol. 2, no. 8, pp. 1804-1820 .

6. S. E. Park, "Epidemiology, virology, and clinical features of severe acute respiratory syndrome-coronavirus-2 (SARS- CoV-2; Coronavirus Disease-19)," Clinical and Experimental Pediatrics,2020, vol. 63, no. 4, pp. 119-124

7. J. S. Kahn and K. McIntosh, "History and recent advances in coronavirus discovery," The Pediatric Infectious Disease Journal, 2005,vol. 24, Supplement, pp. S223-S227 .

8. T. Estola, "Coronaviruses, a New Group of Animal RNA Viruses," Avian Diseases, 1970, vol. 14, no. 2, pp. 330-336.

9. J. Fabricant, "The early history of infectious bronchitis," Avian Diseases, 1998 ,vol. 42, no. 4, pp. 648650 .

10. E. Mahase, Covid-19: First coronavirus was described in The BMJ in 1965, British Medical Journal Publishing Group2020. 
11. E. Kendall, M. Bynoe, and D. Tyrrell, "Virus isolations from common colds occurring in a residential school," British Medical Journal, 1962, vol. 2, no. 5297, pp. 82-86.

12. T. S. Fung and D. X. Liu, "Coronavirus infection, ER stress, apoptosis and innate immunity," Frontiers in Microbiology, 2014, vol. 5, p. 296.

13. V. M. Corman, D. Muth, D. Niemeyer, and C. Drosten,.Hosts and sources of endemic human coronaviruses," in Advances in virus research, Elsevier, 2018 , pp. 163-188.

14. W. Wang, Y., Xu, R. Gao et al., "Detection of SARS-CoV-2 in Different Types of Clinical Specimens," Jama, 2020 vol. 323.

15. K. G. Andersen, A. Rambaut, W. I. Lipkin, E. C. Holmes, and R. F. Garry, "“The proximal origin of SARS-CoV-2,” Nature Medicine, 2020 ,vol. 26, no. 4, pp. 450-452.

16. World Health Organization, Novel Coronavirus (2019- nCoV): situation report, 2020. 5Journal of Diabetes Research

17. Y. Liu, A. A. Gayle, A. Wilder-Smith, and J. Rocklöv, "The Reproductive Number of COVID-19 Is Higher Compared to SARS Corona virus,” Journal of travel medicine, 2020 vol. 27,no. 2.

18. P Mehta, D. F. McAuley, M. Brown, E. Sanchez, R. S. Tatter-sall, and J. J. Manson, . "COVID-19: consider cytokine storm syndromes and immunosuppression," The Lancet, 2020 vol. 395,no. 10229, pp. 1033-1034.

19. World Health Organization, WHO Director-General's opening remarks at the media briefing on COVID-19-11 March2020, Geneva, Switzerland 2020.

20. F. Zhou, T. Yu, R. du et al., "Clinical course and risk factors for mortality of adult in patients with COVID-19 in Wuhan, China: a retrospective cohort study," The Lancet, 2020. vol. 395, no. 10229, pp. 1054-1062.

21. Z. T. Bloomgarden, "Diabetes and COVID-19," Journal of Diabetes, 2020 vol. 12, no. 4, pp. $347-348$.

22. A. Brufsky, "Hyperglycemia, Hydroxychloroquine, and the COVID-19 pandemic," Journal of Medical Virology, 2020 vol. 92, no. 7, pp. 770-775.

23. $73 \%$ of COVID-19 deaths in India are people with comorbidities, no underreporting of deaths: Govt. 2019. https://www.livemint.com/news/india/73- of-covid19-deaths-in-indiaare-people-with-co-morbidities-health-ministry- 11591094600772.html.

24. Bloomgarden ZT. Diabetes and COVID-19. J Diabetes 2020;12:347e8

25. Geerlings SE, Hoepelman AI. Immune dysfunction in patients with diabetes mellitus (DM). FEMS Immunol Med Microbiol 1999;26:259e65.

26. Kaveeshwar SA, Cornwall J. The current state of diabetes mellitus in India. Australas Med J $2014 ; 7: 45 \mathrm{e} 8$.

27. Roncon L, Zuin M, Rigatelli G, Zuliani G ,Diabetic patients with COVID-19 infection are at higher risk of ICU admission and poor short-term outcome. J Clin Virol. 2020, 127:104354.

28. Knapp S. Diabetes and infection: is there a link?-a mini-review. Gerontology. 2013, 59:99-104.

29. Muller LM, Gorter KJ, Hak E, Goudzwaard WL, Schellevis FG, Hoepelman $\mathrm{AI}$, et al. Increased risk of common infections in patients with type 1 and type 2 diabetes mellitus. Clin Infect Dis. 2005, 41:281-8.

30. Hodgson K, Morris J, Bridson T, Govan B, Rush C, Ketheesan N. Immunological mechanisms contributing to the double burden of diabetes and intracellular bacterial infections. Immunology. 2015, 144:171-85.

31. Yang JK, Feng Y, Yuan MY, Yuan SY, Fu HJ, Wu BY, et al. Plasma glucose levels and diabetes are independent predictors for mortality and morbidity in patients with SARS. Diabet Med. 2006, 23:6238 .

32. Guo W, Li M, Dong Y, Zhou H, Zhang Z, Tian C, , et al. Diabetes is a risk factor for the progression and prognosis of COVID-19. Diabetes Metab Res Rev. 2020, e3319.

33. Klonoff DC, Umpierrez GE. COVID-19 in patients with diabetes: risk factors that increase morbidity. Metabolism. 2020, doi: 10.1016/j.metabol.2020.154224.

34. Jinpeng Li, Jianglong Huang, Liang Guo, Rongfen Gao, Kuan Luo, Guang Zeng, Tingbao Zhang, M.eilin Yi, Yihui Huang, Jincao Chen Yibin Yang and Xiaohui Wu, Association Between Diabetes and COVID-19: A Retrospective Observational Study With a Large Sample of 1,880 Cases in Leishenshan Hospital, Wuhan .Frontiers in Endocrinology 2020, Volume 11 Article 478 .

35. Huang I, and Lim MA, Pranata R. Diabetes mellitus is associated with increased mortality and severity of disease in COVID-19 pneumonia - a systematic review, meta-analysis, and meta-regression. Diabetes Metab Syndr. 2020, 14:395-403. 
36. Wu J, Li W, Shi X, Chen Z, Jiang B, Liu J, et al . Early antiviral treatment contributes to alleviate the severity and improve the prognosis of patients with novel coronavirus disease (COVID-19). J Intern Med. 2020, doi: 10.1111/joim.13063.

37. Zhou F, Yu T, Du R, Fan G, Liu Y, Liu Z, et al. Clinical course and risk factors for mortality of adult in patients with COVID-19 in Wuhan, China: a retrospective cohort study. Lancet. 2020, 395:105 .

38. Akin OsibogunID, Mobolanle Balogun, Akin Abayomi,, Jide Idris, Yetunde KuyinuID, Oluwakemi OdukoyaID, Ololade Wright, Remi Adeseun, Bamidele Mutiu, Babatunde Saka, Nike Osa, Dayo Lajide, Ismael Abdus-Salam, Bodunrin Osikomaiya, Oluwatosin OnasanyaID, Bisola Adebayo, Yewande Oshodi, Sunday Adesola, Olu Adejumo, Olufemi ErinosoID, Hussein Abdur-Razzaq, Abimbola Bowale, Kingsley Akinroye. Outcomes of COVID-19 patients with comorbidities in southwest Nigeria PLoS ONE 2020 ,16(3): e0248281. 